Research Article

\title{
Monitoring Regional Urban Dynamics Using DMSP/OLS Nighttime Light Data in Zhejiang Province
}

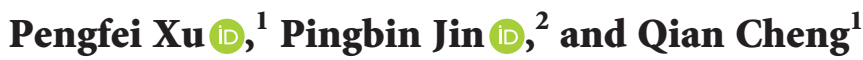 \\ ${ }^{1}$ Zhejiang Gongshang University, College of Tourism and City, Jiaogong Road 149, Hangzhou 310035, China \\ ${ }^{2}$ Zhejiang University, School of Earth Science, Yuhangtang Rd 866, Hangzhou 310027, China \\ Correspondence should be addressed to Pingbin Jin; chshs@zju.edu.cn
}

Received 3 December 2019; Revised 13 March 2020; Accepted 4 May 2020; Published 31 May 2020

Academic Editor: Rafał Stanisławski

Copyright (c) 2020 Pengfei Xu et al. This is an open access article distributed under the Creative Commons Attribution License, which permits unrestricted use, distribution, and reproduction in any medium, provided the original work is properly cited.

Accurate monitoring of urban regions and urban sprawls is critical to the detection and assessment of regional development. The nighttime light images of Defense Meteorological Satellite Program's Operational Linescan System (DMSP/OLS) provide us direct solutions to make spatial descriptions of urban regions. Unfortunately, accurate monitoring of urban regions is apt to be hampered due to the shortages of the DMSP/OLS data. In this study, we utilized a new urban region extraction strategy based on the edge-detection method which is widely applied in automatic digital image processing. The edges of urban areas in Zhejiang province were identified based on the distributions and values of pixels. Compared with other traditional methods, the urban regions extracted in this study present a higher overall accuracy and kappa coefficient (OA $=93.1409 \%$; Kappa $=0.8755)$. Two periods of the urban dynamic process and urban sprawl pattern in Zhejiang from 1992-2013 were further detected by the proposed method. At city level, the drastic increase in urban areas was found in cities of Hangzhou and Ningbo. This study provides an objective and convenient solution to the accurate identification of urban regions, which is also an important step to better understand the urban dynamics and urban development.

\section{Introduction}

The dynamic changes of urban areas as well as the distributions of urban regions are essential criteria in government decision-making about urban development planning. According to the National Bureau of Statistics of China, the urbanization rate of Mainland China in 2017 has increased nearly 3 times to $58.52 \%$ compared with that in 1978 (http:// www.stats.gov.cn/). Great changes have taken place with the rapid increase in urban sprawl. Acquiring accurate information about the urban sprawl and urban dynamics at different levels is essential to the better understanding of urban development.

Traditional urbanization studies are mostly based on statistic data collected by the government, which is usually lack of timeliness and accuracy and thus could not provide timely spatial detection of urbanization. Fortunately, the techniques of remote sensing show better results in detecting urban sprawls and urban regions since they can provide much more direct and frequent temporal coverage of the study regions $[1,2]$. Various remote sensing data have been applied at different scales with different research targets such as the Landsat Thematic Mapper/Enhanced Thematic Mapper plus (TM/ETM+), QuickBird, IKONOS, and SPOT/ High Resolution Visible (HRV). Although these datasets present high or medium spatial resolutions, they are less popular in long-time detection of regions with large scales due to the relatively high cost, complicated data processing procedure, and high equipment requirements. Compared with those images, the nighttime light image of Defense Meteorological Satellite Program's Operational Linescan System (DMSP/OLS) is more economical and is available over a larger temporal window $[3,4]$. It seems to be a better data source for monitoring urban regions with large scales and long histories.

The DMSP/OLS images record nighttime light emitted from the earth's surface which is derived from a variety of natural and man-made phenomena, such as those from 
human settlements and gas flares. The nighttime light would not be affected by shadows, vegetation covers, and other interference factors $[5,6]$. Although the application of the data has been greatly limited due to the shortages of blooming, over flow, and loss of calibration [7, 8], it can provide both light brightness and geospatial information of the human activities, which has been proved to be a valuable source for both the natural and social sciences detection [9-11]. Moreover, in 1992, the open access digital archive was established by the National Oceanic and Atmospheric Administration/National Geophysical Data Center (NOAA/ NGDC). Since then, the DMSP/OLS nighttime images can be acquired freely and timely [12], which leads to many related research studies, such as population density detecting, urbanization process monitoring, regional GDP estimating, electricity consumption modeling, and so on [13-18].

The DMSP/OLS nighttime light images consist of pixels with locations and illuminance information of ground surface, and it had been widely accepted in urbanizationrelated research studies, especially the detections related with spatial information $[19,20]$. However, most of the previous studies are limited by the accurate extraction of urban regions from lighted pixels. The urban region extraction method was firstly proposed by Imhoff based on a threshold method [21]. With the initial effort of Imhoff, the official census data-based methods and scale-based thresholds were subsequently proposed [22-24]. However, these threshold-related methods often omit small proportional settlements or overestimate urban extents [17]. Fortunately, based on the traditional solutions of remote sensing image classification, some semiautomatic-based detection methods were derived, such as the marker-controlled watershed segmentation method, neighborhood statistics analysis method, and support-vector machine- (SVM-) based detection supervised and unsupervised classification methods. These methods provide us relatively objective and accurate solutions to identify urban areas, but they have not been widely accepted since the data calculation process is relatively complicated and labor intensive [25-28].

In order to monitor urban dynamics and extract urban regions accurately and quickly, an edge-detection-based method using the consequential Sobel operator was applied in this study. The Sobel operator was first proposed by Irwin Sobel [29]; with the selected filter, the operator can not only collect information of edges but also keep detailed information of images. Thus, it is quite prominent in information technology fields such as machine learning, digital media, and computer vision [30-32]. Compared with the aforementioned methods, the Sobel operator can automatically recognize and understand image features with the detected edges and is not limited by other data sources [33]. Therefore, in this study, pixel-based identification using the Sobel operator was applied to map urban areas. Moreover, to validate the effect of the proposed method in urban region mapping, the long-term urban dynamics and urban sprawl of Zhejiang province were further detected.

This study aims to extract urban regions and detect urban dynamics accurately and effectively at regional level using the DMSP/OLS nighttime light images. The Sobel edge detection operator was applied to extract urban regions; comparative analyses with other widely used urban extraction methods mentioned above were also performed. In addition, the long-term urban regions and urban sprawl dynamics of Zhejiang province were detected.

\section{Methodology}

\subsection{Study Area and Data}

2.1.1. Study Area. Zhejiang province, located in the east of China, is an important part of the urban agglomeration in the Yangtze River Delta and is therefore the subject of this study. The Yangtze River Delta is one of the biggest urban agglomerations around the world, of which Zhejiang province occupies a total of 105.5 thousand $\mathrm{km}^{2}$. In the past few decades, Zhejiang experienced a fast socioeconomic and urban development. According to the National Bureau of Statistics, the population of Zhejiang has grown to nearly 50 million, showing an increase of more than 15\% compared with that in 1990s. The total gross domestic product (GDP) of Zhejiang in 2010 increased nearly 30 times over 1990s, from 90.4 billion Yuan (in 1990) to more than 2774.7 billion Yuan (in 2010).

Although the socioeconomic growth is rapid in Zhejiang province, it is regional-dependent. The western and inland areas are mostly mountainous, while the plains are concentrated in the eastern and coastal areas (Figure 1(a)). Also, making the regional development of inland areas slower than coastal areas leads to significant differences of regional GDP and population distribution (Figures 1(b) and 1(c)). So, we choose Zhejiang province as the study region in this analysis.

2.1.2. Data and Data Processing. The annual stable DMSP/ OLS nighttime light images of version 4 from 1992-2013 and land use and land cover change (LUCC) data of 2010 Zhejiang province were used in this study. DMSP/OLS data can be obtained from NOAA/NGDC (https://www.ngdc. noaa.gov), and LUCC data were downloaded from the Resource and Environment Data Cloud Platform of Chinese Academy of Sciences (REDCP, CAS) (http://www.resdc.cn). Apart from these, grid format of population and GDP data of Zhejiang province in 2010 were also acquired from the REDCP, CAS, and auxiliary data related to province and cities boundaries.

Since the DMSP/OLS nighttime light images applied in this research were acquired from different satellites, covering years from 1992 to 2013, the second-order polynomial regression model was applied firstly to calibrate the images [34]:

$$
D N_{\mathrm{cal}}=a+b * D N+c * D N^{2}
$$

where $D N_{\text {cal }}$ is the calibrated value of $D N$ and $a, b$, and $c$ are coefficients of the regression model.

Moreover, DMSP/OLS and LUCC are with respective different spatial resolutions and produced under different 


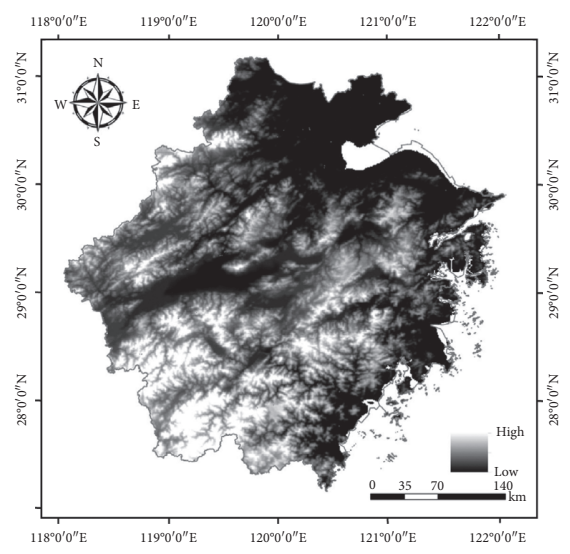

(a)

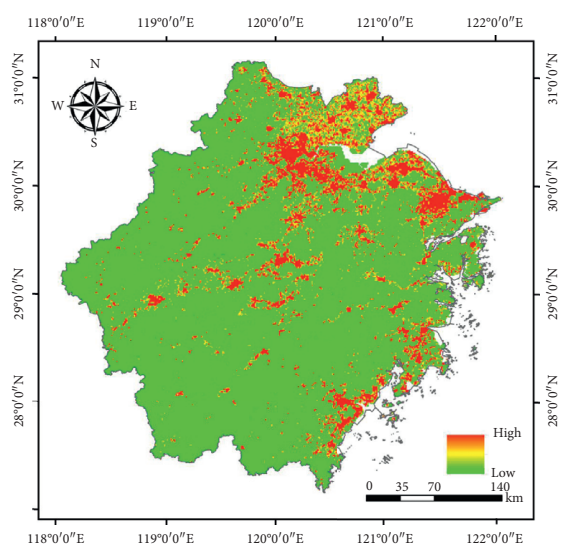

(b)

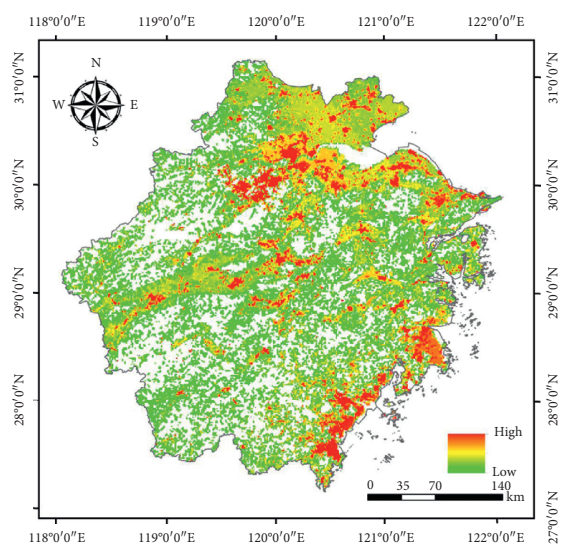

(c)

Figure 1: (a) The distribution of topography, (b) regional GDP distribution, and (c) population distribution of Zhejiang.

coordinate systems. Therefore, all of them were projected firstly using the WGS84 geography coordinate system and resampled into $1 \mathrm{~km}$ for further comparisons. After that, they were also clipped according to the boundaries of Zhejiang province and cities for further analyses.

2.2. Urban Region Extraction. In this study, the urban regions were defined according to the characteristics of light emanation from DMSP/OLS nighttime images. Also, based on the $D N$ values and distance from pixels, the DMSP/OLS nighttime images were firstly divided into 3 consecutive parts, namely, potential rural regions, potential urban transition regions, and potential urban centers. In rural and urban center regions, pixels are always with low or high $D N$ values and are relatively stable, while the pixel values in transition regions always locate between urban centers and rural regions, cover a wide range of $D N$ values, and also experience drastic changes [35-37].

To define boundaries of each part from the nighttime images, a step-by-step flowchart was developed (Figure 2). First, we extract potential nonurban pixels and urban center pixels of the selected regions using the threshold method which has been widely applied in previous studies. Then, a Sobel edge operator was applied to the transition regions to calculate the gradient distributions of each pixel. With the detected gradient, edges of urban regions within transition areas can be identified; together with the extracted urban centers, urban regions of selected study areas can be mapped. Moreover, an accuracy assessment was further made to test the extraction results of the proposed method.

2.2.1. Transition Region Identification. To extract urban regions, we need to define the scope of transition regions in which the urban boundaries locate in. In this step, the generally used threshold method was employed, and the $D N$ value of 30 and 60 were chosen as urban boundaries based on empirical analysis from previous detections [14, 21, 24, 27]. So, in this study, the $D N$ value of 30 and 60 were chosen as initial limits to distinguish transition regions from rural and urban centers.

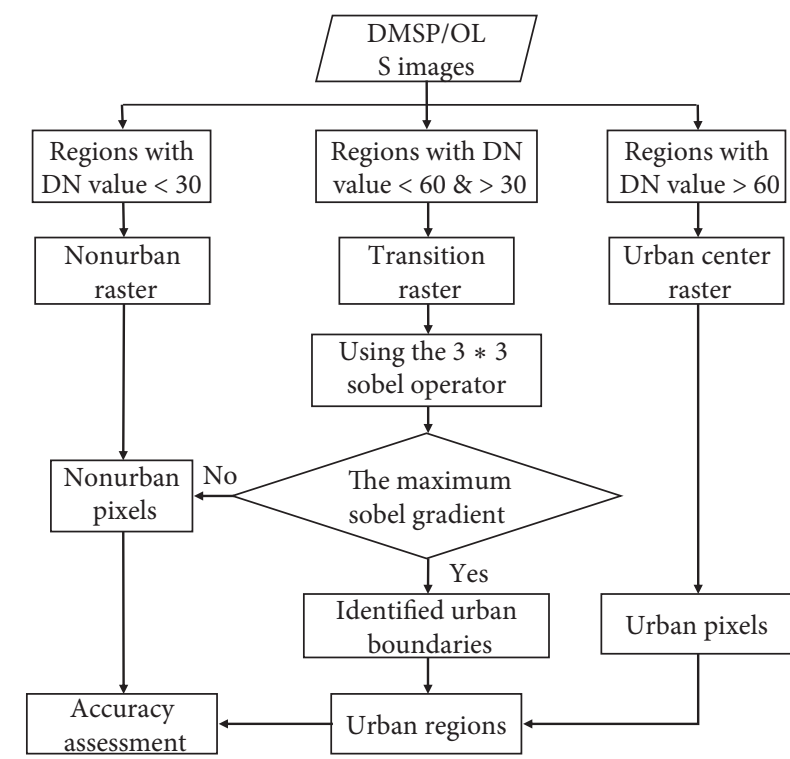

Figure 2: Flowchart of the urban extraction method.

2.2.2. Application of the Sobel Operator. The Sobel edge operator performs a $2 \mathrm{D}$ spatial gradient measurement on the images and is a well-known solution applied in image edge detection in various research fields [32]. The Sobel operator consists of $23 * 3$ moving-window kernels of different directions; the gradient results measure how rapid pixels values are changing with distance in two perpendicular directions and also the difference with other surrounding pixels $[29,38]$; in addition, they can also be combined together to calculate the gradient $(G)$ at each pixel and even the direction of the gradient [39]. Using the Sobel edge detection method discussed aforementioned, the gradients of each pixel within transition regions can be calculated.

2.2.3. Urban Region Identification. The gradient of pixels within transition regions can be calculated with the Sobel operator, and each gradient value represents the difference with the other 8 surrounding pixels within the nighttime 
images. The lower the gradient value is, the higher uniformity the $3 * 3$ convolution kernels (representing $9 \mathrm{~km}^{2}$ ) have. In the gradient distribution of DMSP/OLS nighttime images, the value experienced multiple changes when the distance from urban centers decreases. Pixels gradients close to the defined regions of rural and urban centers are relatively smaller than the transition regions because of the higher uniformity. Based on the above analysis, we assumed that pixel distributions of region boundaries are quite different and the gradients are thus larger. So, in this study, the gradient value of local maximum was considered as the identified boundary to separate rural and urban areas within transition regions. Therefore, the urban areas of study regions can be identified.

There are no direct urban region products which can be applied to validate the accuracy of our detection; so, in this study, the widely accepted LUCC data were selected to provide ground surface information of Zhejiang province. Overall accuracy and the Kappa coefficient were used as statistical criteria.

\section{Result}

3.1. Urban Regions Detected in Zhejiang Province. With the proposed method, urban regions of Zhejiang province from 1992 to 2013 were mapped. The result indicated that great urban development has taken place in the past few decades, significant regional difference of urban sprawl also occurred, and the urban development in the north and coast areas were much more active than inland regions as shown in (Figure3).

\subsection{Accuracy Assessment of the Proposed Method. To assess} the superiority of our proposed method in urban region identification, a few widely used strategies were selected to make comparative analyses: mutation detection, scale-based threshold method, neighborhood statistics analysis, and stratified SVM-classification method. Meanwhile, the LUCC image of Zhejiang province was chosen as ground truth. The assessment results in Figure 4 and Table 1 indicate that the edge-detection method applied in this study is much more accurate and convincing in urban region identification compared with other methods, with higher overall accuracy and kappa coefficient $(\mathrm{OA}=93.1409 \%$; Kappa $=0.8755)$, followed by the stratified SVM-classification method and scale-based threshold method, where these two methods are always computationally intensive and the processes are quite complicated.

\subsection{Long-Term Urban Dynamics of Zhejiang Province.} With the edge-detection method in this study, urban regions can be extracted. Based on the extraction results, we further analyzed urban region dynamics of Zhejiang province from 1992 to 2013. Figure 5(a) demonstrates the dynamics of urban region pixels and the annual increase of urban pixels within detected regions. During the study period of 1992 to 2013, the urban areas of Zhejiang increased a lot and the increase trend was relatively steady with smaller annual increase from 1992-2013. However, after the turning point of 2003, the speed of urban dynamics increased rapidly and the annual increase was larger than before. Figure 5(b) shows the urban sprawl pattern of Zhejiang province from 1992 to 2013; the distribution and characteristics of urban regions were detected. We found that the urbanized regions during the study period of Zhejiang mainly located in the north and coastal areas of the province, which provide us a quick and direct description of urban development.

3.4. Feasibility of City-Level Detection. Previous research found that the extraction accuracy of other methods is related to the level of urban development. However, in this study, we performed city-level extraction to verify the effectiveness of the proposed edge-detection method; cities with different regional characteristics and development levels in Zhejiang Province were chosen (the island of Zhoushan was ignored in this analysis because the size is relatively small and the night light phenomenon is easily disturbed by light blooming which might obscure the detecting result).

Figure 6 shows the city-level extraction results of urban regions, and in Table 2, official socioeconomic indicators related with urban development levels and accuracy assessment results can be seen. Together with the comparative study of extraction results in Figure 6, it is clear that the OA and Kappa are quite high within highly developed cities such as Hangzhou and Wenzhou (with high level of population and per capita GDP), and in less developed cities (Population and per capita GDP are relatively small) of Lishui and Quzhou, the same results can also be found. It is surprised that, in the highly developed city of Jiaxing, the detected OA and Kappa are unexpectedly smaller than others. Hence, we believe that the method in this paper can achieve effective extraction result at the city level, and the extraction accuracy has nothing to do with the level of urban development, which is superior to other methods.

\subsection{City-Level Urban Development of Zhejiang Province.} With the detected urban regions from DMSP/OLS nighttime images, the sprawl of urban areas in each city of Zhejiang was also detected in Figure 7. Each city underwent an urban region expansion during the study period and presented differently in urban dynamics. In cities of Hangzhou and Ningbo, the detected urban regions are larger than other cities and drastic increase of urban regions were also found, while in inland cities such as Quzhou and Lishui, both the urban regions and urban dynamics were smaller.

\section{Discussion}

4.1. Priorities of the Edge-Based Method. The DMSP/OLS nighttime light images provide us direct descriptions of the night scene as for land surface, and a large number of methods have been applied to extract urban areas from it [40]. Compared with the traditional threshold-based and semiautomatic methods in previous research studies, the edge-detection method has the following advantages. First, the proposed method mainly depends on the brightness and 


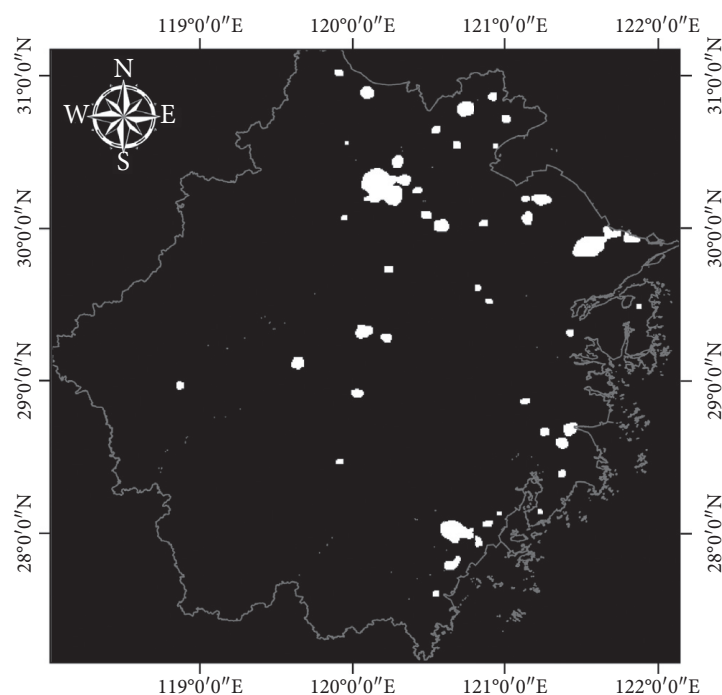

(a)

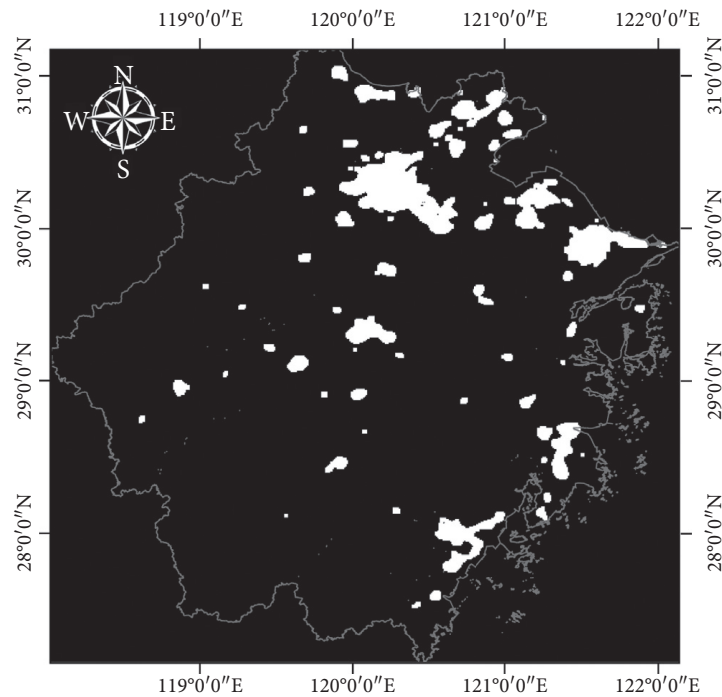

(c)

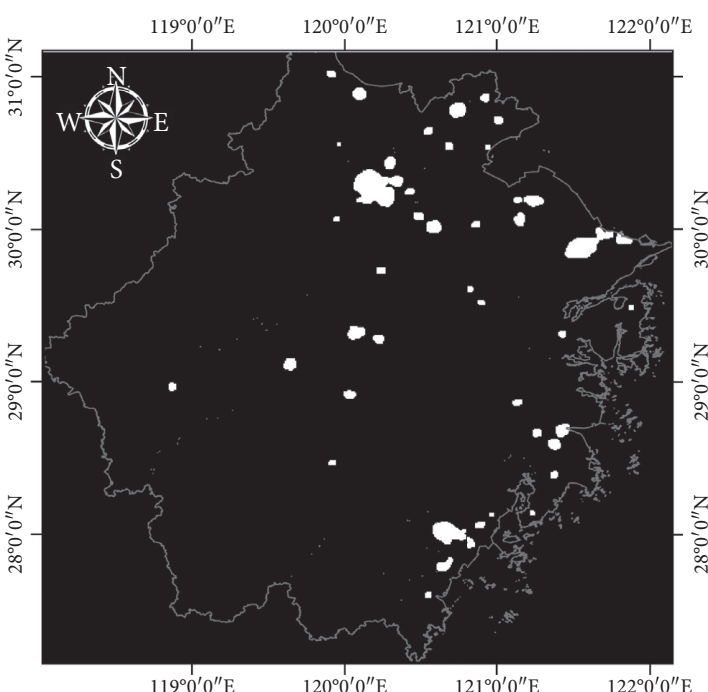

(b)

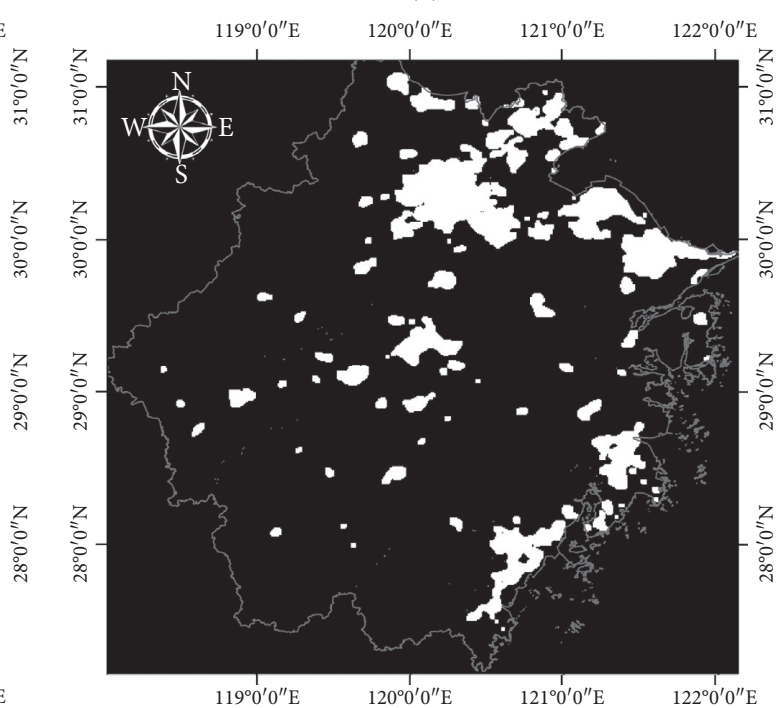

(d)

FIgURE 3: Extracted urban regions of Zhejiang province. (a) 1992, (b) 2000, (c) 2007, and (d) 2013.

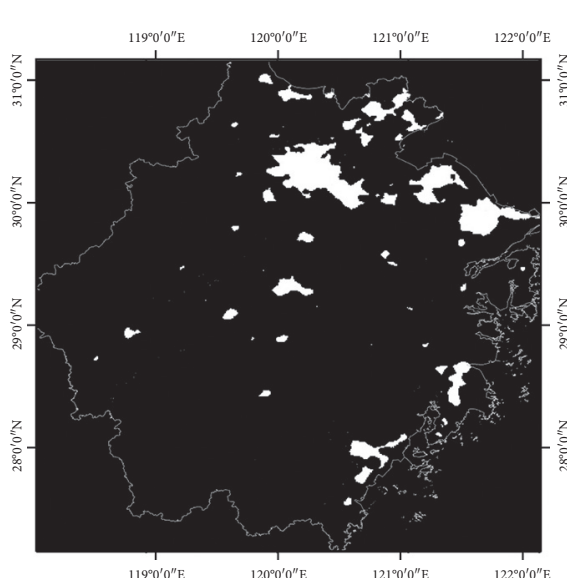

(a)

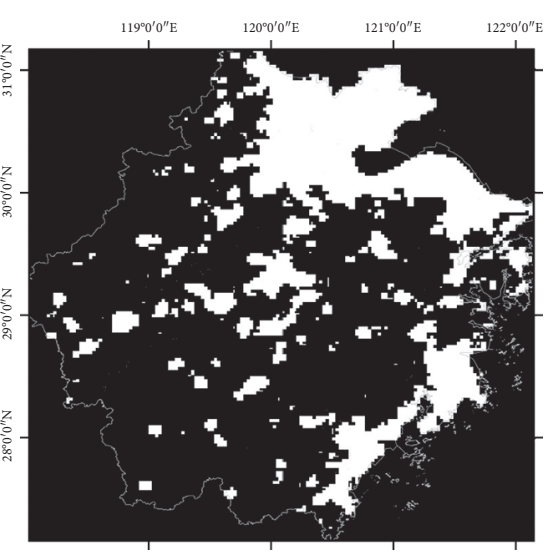

(b)

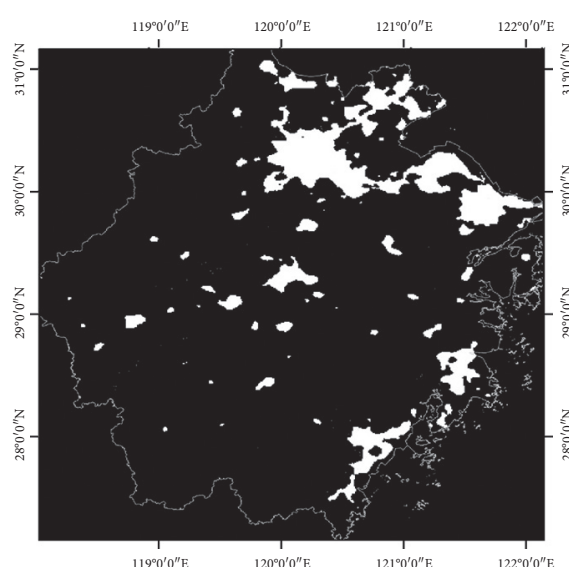

(c)

Figure 4: Continued. 


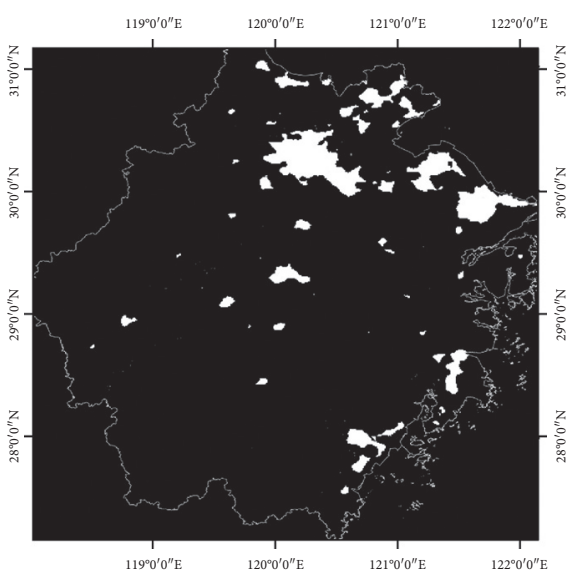

(d)

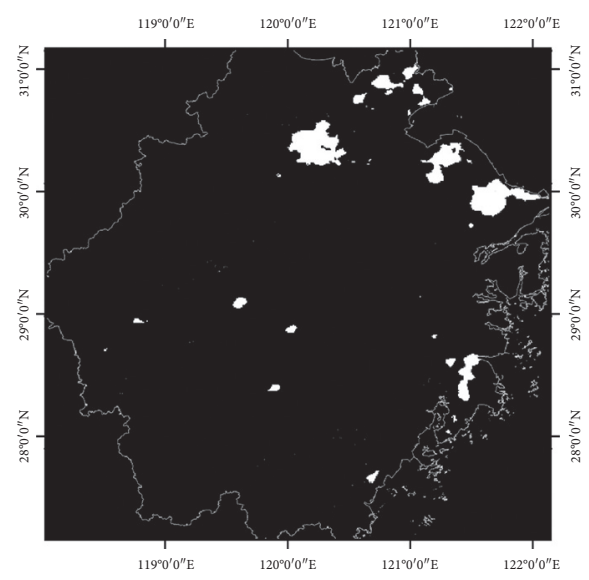

(e)

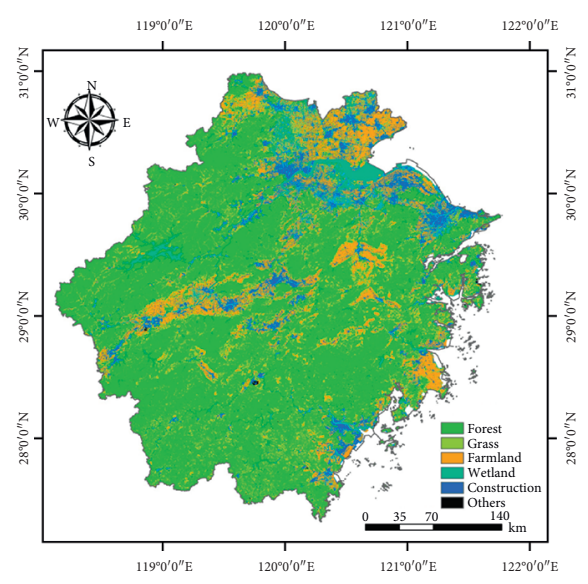

(f)

Figure 4: Urban extraction results of Zhejiang province in 2010 using the Sobel edge detection method (a), NSA method (b), SVM method (c), mutation detection method (d), Scale-based threshold method (e), and land use and land cover change (f) of Zhejiang province in 2010.

TABLE 1: Overall accuracy and kappa coefficient of different extraction methods.

\begin{tabular}{lcr}
\hline Method & Overall accuracy (\%) & Kappa coefficient \\
\hline Mutation detection & 83.7427 & 0.6892 \\
Scale-based threshold & 90.5186 & 0.8514 \\
NSA & 85.7964 & 0.7626 \\
Edge detection & 93.1409 & 0.8755 \\
SVM & 89.3865 & 0.7830 \\
\hline
\end{tabular}

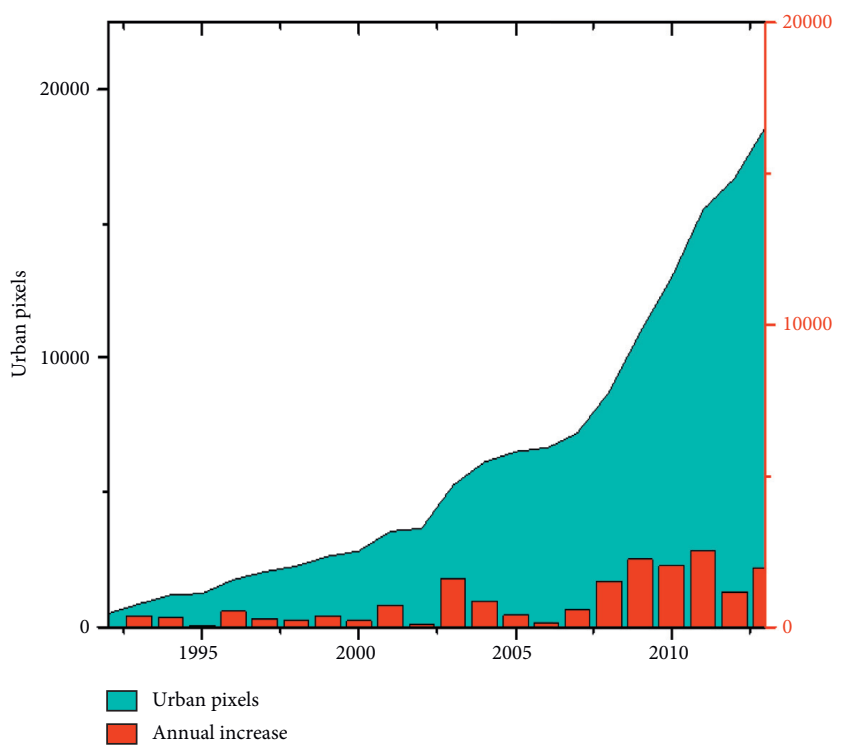

(a)

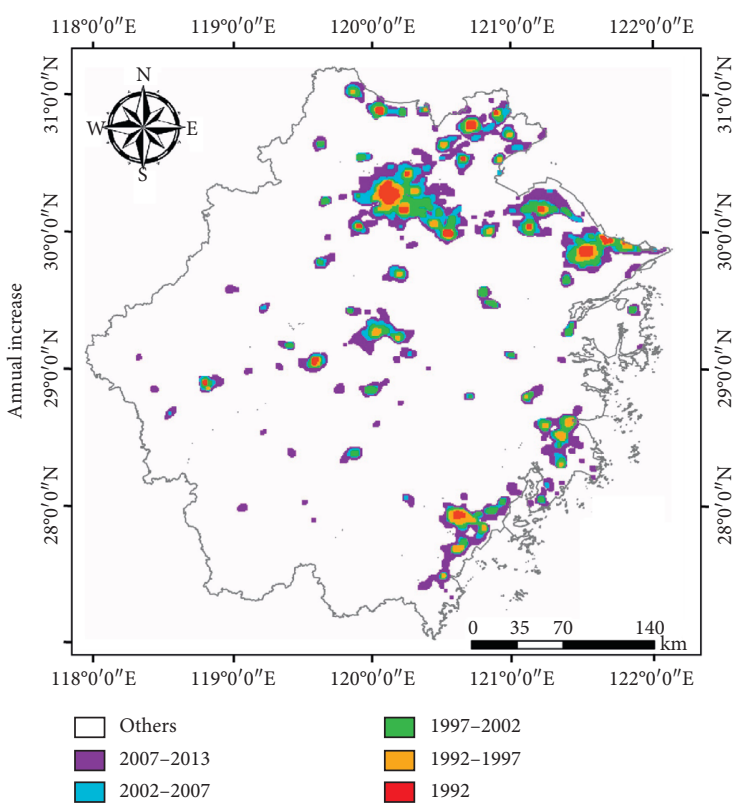

(b)

Figure 5: (a) Dynamics of extracted urban pixels and (b) distribution of urban sprawl of Zhejiang province.

distribution characteristics of pixels and does not rely on statistical data, so it is not affected by the accuracy and timeliness of statistics data [41]. Second, the urban areas were identified based on the value and regional characteristics of pixels in the proposed method, the process is relatively objective, and the result can be more approximate to the real distributions [42]. Moreover, the proposed method operates a separable and integer-valued $3 * 3$ filter in 

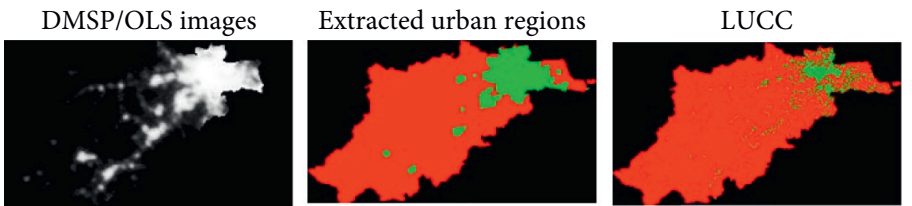

Hangzhou $\mathrm{OA}=94.1412 \%$

Kappa $=0.8911$
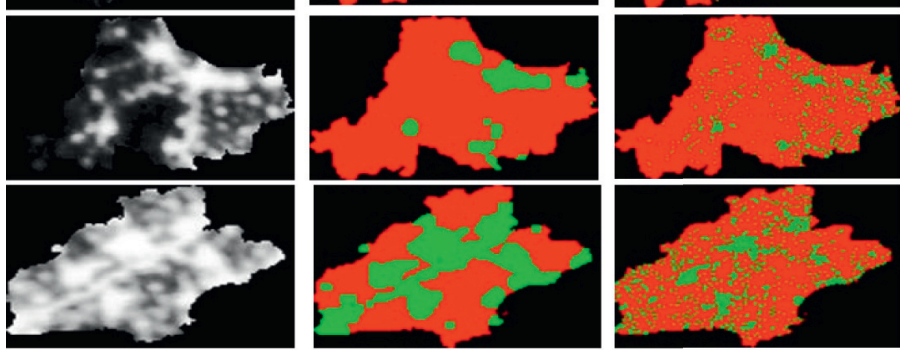

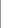

Huzhou

$\mathrm{OA}=90.3974 \%$

Kappa $=0.8286$
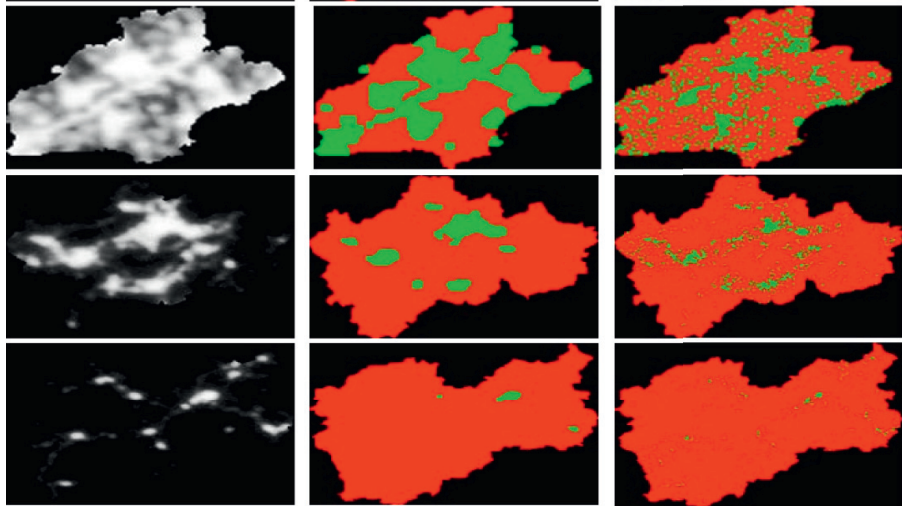

Jiaxing

$\mathrm{OA}=79.8020 \%$

Kappa $=0.6793$
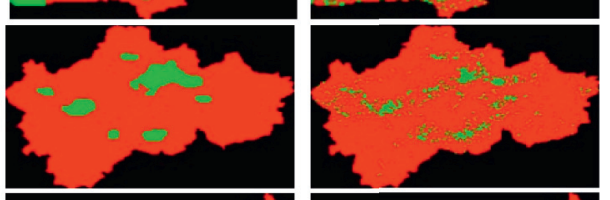

Kappa $=0.6793$
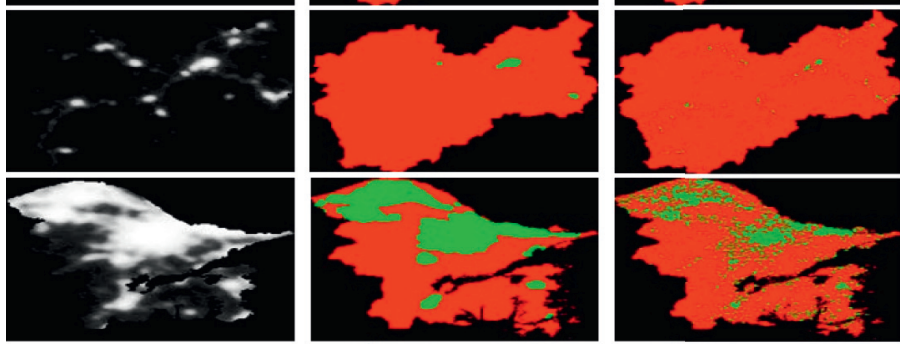

Jinhua

$\mathrm{OA}=93.4396 \%$

Kappa $=0.8787$
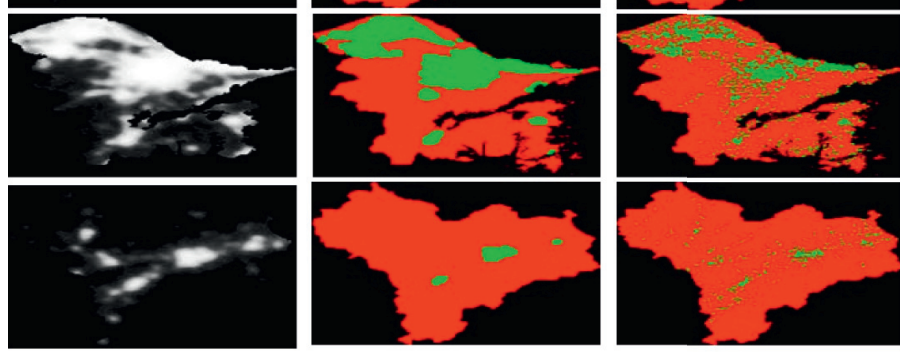

Lishui

$\mathrm{OA}=98.0914 \%$

Kappa $=0.9611$
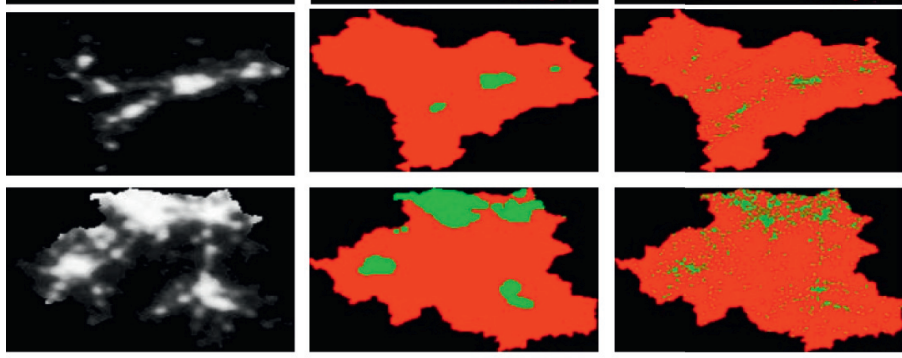

Ningbo

$\mathrm{OA}=86.9175 \%$

Kappa $=0.7806$
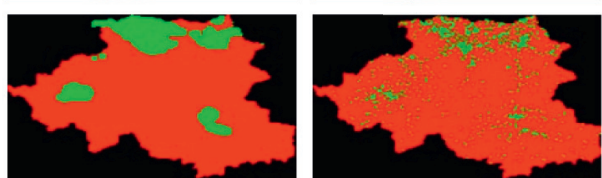

Quzhou

$\mathrm{OA}=96.6188 \%$

Kappa $=0.9347$
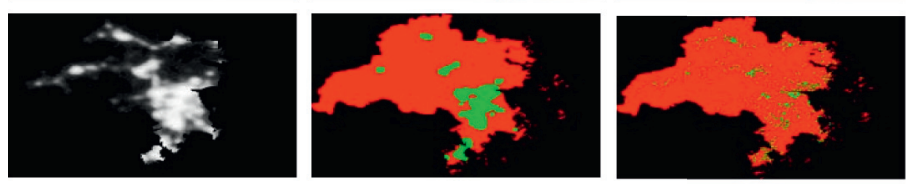

$\mathrm{OA}=94.4976 \%$

Kappa $=0.8815$
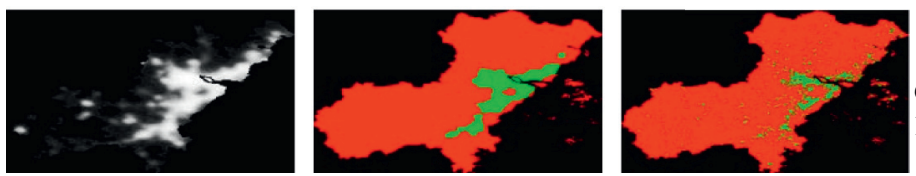

Wenzhou

$\mathrm{OA}=94.6773 \%$

Kappa $=0.8989$

FIgURE 6: City-level urban extraction result within Zhejiang province.

TABLE 2: Socioeconomic development and extraction accuracy of different cities.

\begin{tabular}{lccc}
\hline City & Population (10000 persons) & Per capital GDP (yuan) & OA (\%) \\
\hline Hangzhou & 689.12 & 86691 & 94.1412 \\
Huzhou & 259.98 & 50149 & 90.3974 \\
Jiaxing & 341.60 & 67534 & 79.8020 \\
Jinhua & 466.65 & 45031 & 93.4396 \\
Lishui & 259.65 & 24913 & 98.8911 \\
Ningbo & 574.08 & 90175 & 0.6793 \\
Quzhou & 251.24 & 30153 & 0.8787 \\
Shaoxing & 438.91 & 63770 & 0.9611 \\
Taizhou & 583.14 & 41777 & 96.6188 \\
Wenzhou & 786.80 & 37359 & 90.5757 \\
\hline
\end{tabular}




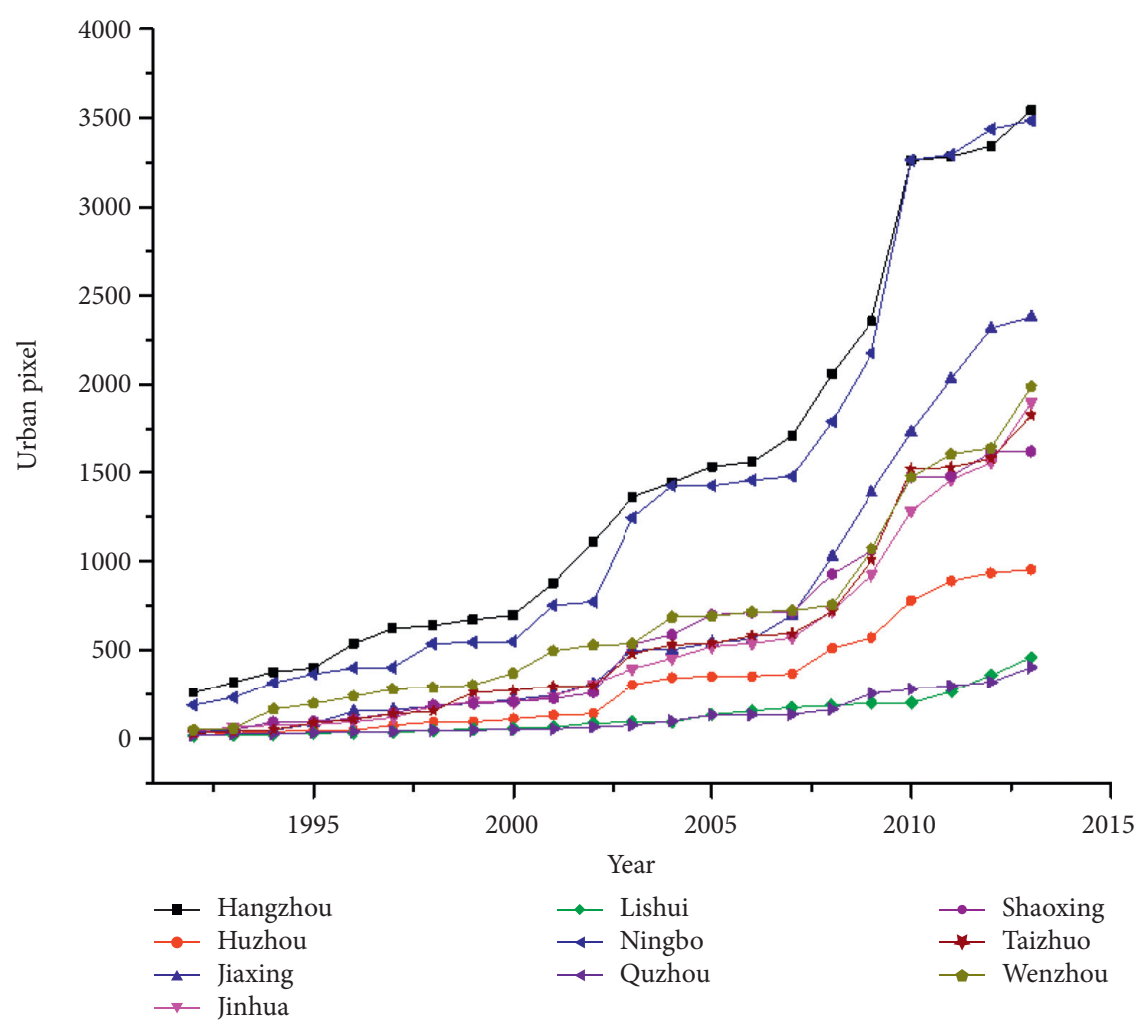

FIgURE 7: City-level urban sprawl of Zhejiang province.

two orthogonal directions and is relatively convenient and quick in processing, especially when comparing with other existing semiautomatic methods [38]. Finally, the edgedetection methods show better accuracies in urban region detection compared with previous methods with higher overall accuracy and Kappa coefficient.

4.2. Factors Related with Detection. In Figure 6, we can find that urban regions within cities with different levels of development were extracted and the edge-detection method was quite effective in different cities with high value of OA and Kappa coefficient. However, the result in the city of Jiaxing was different, and a further analysis of the characteristics and distributions of pixels within Jiaxing was made. We found three main reasons to explain the relatively low overall accuracy and Kappa coefficient of the Jiaxing city. First, the nighttime images cannot effectively reflect the light phenomenon within the city. As a well-known city of rivers and lakes, nearly $10 \%$ of the land surfaces in Jiaxing are covered by water, and the situation of light reflection in water regions are quite drastic which caused the light blooming of adjacent pixels (this phenomenon is quite common in NTL images, especially the water regions). Blooming is the basic feature of DMSP/OLS images in which the DN values recorded in a pixel are equal to the light of real brightness plus the reflected light from nearby pixels, so the ground truth of light brightness is exactly smaller than the light recorded on the applied images [43]. Second, the complexity of DN-value distribution was analyzed. As we can see in Figure 6, the DN value of Jiaxing is relatively high, with an average of 19.43 which is much larger than the average value of Zhejiang province (6.88). These highbrightness areas are scattered and are with small areas, which makes the Sobel operator difficult to distinguish exact boundaries of urban and rural; therefore, the ability of our method was limited. Finally, the low sensitivity of Sobel in the fragmentized area was determined. The Sobel operator extracts features from the ground surface, and its $3 * 3$ convolution kernel might soothe the input image to a greater extent, making the operator less sensitive to distinguish, especially in mixed areas, and the result might be imprecise [32].

4.3. Limitations and Further Research. The proposed edgedetection method provided an accurate solution to extract urban regions from nighttime light images; however, there are a few limitations need to be concerned. For example, the detection results obtained with this method are always in a binary format, and further processing is needed if we want to acquire more information about the city lights. Moreover, the night images applied in this study are in coarse resolution and the phenomenon of saturation and blooming exists; to acquire accurate information of features of urban, finer resolution images are required. Besides, acquiring urban regions using the nighttime light images only is still weak in reliability compared with other finer resolution data sources; hence, in the future studies, we might test the automatic extraction results combined with other remote sensing data. 


\section{Conclusion}

With the edge-detection operator, we proposed a much more accurate and convenient method to automatically extract urban areas from the DMSP/OLS nighttime light images. We found that the applied method was quite effective to identify boundaries of rural and urban with high value of OA compared with traditional methods. Moreover, based on the proposed method, we successfully detected the urban dynamics and urban sprawl pattern at provincial and city level from 1992 to 2013 and discovered that the proposed method was effective to distinguish urban areas from regions with different developing levels which are superior to other methods. The proposed method significantly overcomes the limitations of traditional methods such as statistics-based evaluation and large data volume and provides a quick and objective judgment of urban areas; it is beneficial to monitoring of urban dynamics and urban sprawls.

\section{Data Availability}

The tif data used to support the findings of this study can be freely downloaded from the website of NGDC (https://www. ngdc.noaa.gov/).

\section{Conflicts of Interest}

The authors declare that they have no conflicts of interest.

\section{Acknowledgments}

The first author appreciates the support from the National Key Research and Development Program (2017YFB0503902) and the China Scholarship Council (201706320300) for his joint PhD Scholarship.

\section{References}

[1] S. Angel, J. Parent, D. L. Civco, A. Blei, and D. Potere, "The dimensions of global urban expansion: estimates and projections for all countries, 2000-2050," Progress in Planning, vol. 75, no. 2, pp. 53-107, 2011.

[2] H. Huang, Y. Chen, N. Clinton et al., "Mapping major land cover dynamics in Beijing using all Landsat images in Google Earth Engine," Remote Sensing of Environment, vol. 202, pp. 166-176, 2017.

[3] K. Yi, H. Tani, Q. Li et al., "Mapping and evaluating the urbanization process in Northeast China using DMSP/OLS nighttime light data," Sensors, vol. 14, no. 2, pp. 3207-3226, 2014.

[4] Q. Zhang and K. C. Seto, "Mapping urbanization dynamics at regional and global scales using multi-temporal DMSP/OLS nighttime light data," Remote Sensing of Environment, vol. 115, no. 9, pp. 2320-2329, 2011.

[5] T. A. Croft, "Nighttime images of the earth from space," Scientific American, vol. 239, no. 1, pp. 86-98, 1978.

[6] C. D. Elvidge, K. E. Baugh, M. Zhizhin, and F.-C. Hsu, "Why VIIRS data are superior to DMSP for mapping nighttime lights," Proceedings of the Asia-Pacific Advanced Network, vol. 35, pp. 62-69, 2013.
[7] P. Kumar, H. Sajjad, P. K. Joshi et al., "The luminous intensity of Beijing, China using DMSP-OLS night-time lights series data for estimating population density," Physics and Chemistry of the Earth, Parts A/B/C, vol. 109, pp. 31-39, 2019.

[8] P. Singh, S. Rehman, H. Sajjad, B. R. Tripathy, M. Rani, and S. Singh, "Analyzing trend in artificial light pollution pattern in India using NTL sensor's data," Urban Climate, vol. 27, pp. 272-283, 2019.

[9] X. Li, C. Elvidge, Y. Zhou, C. Cao, and T. Warner, "Remote sensing of night-time light," International Journal of Remote Sensing, vol. 38, no. 21, pp. 5855-5859, 2017.

[10] J. Proville, D. Araiza, and G. Wagner, "Night-time light: a global, long term look at links to social-economic trends," PLoS One, vol. 12, no. 3, Article ID e0174610, 2017.

[11] X. Xin, L. Bin, and D. Kaichang, "Monitoring urban expansion using time series of night-time light data: a case study in Wuhan, China," International Journal of Remote Sensing, vol. 21, no. 38, pp. 6110-6128, 2017.

[12] Q. Huang, X. Yang, B. Gao, Y. Yang, and Y. Zhao, "Application of DMSP/OLS nighttime light images: a meta-analysis and a systematic literature review," Remote Sensing, vol. 6, no. 8, pp. 6844-6866, 2014.

[13] T. R. Chand and K. V. S. Badarinath, "Spatial characterization of electrical power consumption patterns over India using temporal DMSP-OLS night-time satellite data," International Journal of Remote Sensing, vol. 3, no. 30, pp. 647-661, 2009.

[14] Y. Ju, I. Dronova, Q. Ma, and X. Zhang, "Analysis of urbanization dynamics in mainland China using pixel-based night-time light trajectories from 1992 to 2013," International Journal of Remote Sensing, vol. 38, no. 21, pp. 6047-6072, 2017.

[15] H. Letu, M. Hara, H. Yagi et al., "Estimating energy consumption from night-time DMPS/OLS imagery after correcting for saturation effects," International Journal of Remote Sensing, vol. 31, no. 16, pp. 4443-4458, 2010.

[16] Q. Li, L. Lu, Q. Weng, Y. Xie, and H. Guo, "Monitoring urban dynamics in the southeast U.S.A. using time-series DMSP/ OLS nightlight imagery," Remote Sensing, vol. 8, no. 7, p. 578, 2016.

[17] D. Lu, H. Tian, G. Zhou, and H. Ge, "Regional mapping of human settlements in Southeastern China with multisensor remotely sensed data," Remote Sensing of Environment, vol. 112, no. 9, pp. 3668-3679, 2008.

[18] Y. Zhou, T. Ma, C. Zhou, and T. Xu, "Nighttime light derived assessment of regional inequality of socioeconomic development in China," Remote Sensing, vol. 7, no. 2, pp. 12421262, 2015.

[19] T. Ma, C. Zhou, T. Pei, S. Haynie, and J. Fan, "Quantitative estimation of urbanization dynamics using time series of DMSP/OLS nighttime light data: a comparative case study from China's cities," Remote Sensing of Environment, vol. 124, pp. 99-107, 2012.

[20] T. Xu, T. Ma, C. Zhou, and Y. Zhou, "characterizing spatiotemporal dynamics of urbanization in china using time series of DMSP/OLS night light data," Remote Sensing, vol. 6, no. 8, pp. 7708-7731, 2014.

[21] L. Imhoff, W. T. Lawrence, D. C. Stutzer, and C. D. Elvedge, "A technique for using composite DMSP/OLS "City Lights" satellite data to map urban area," Remote Sensing of Environment, vol. 61, no. 3, pp. 361-370, 1997.

[22] M. Hendenson, E. T. Yeh, P. Gong, C. Elvidge, and K. Baugh, "Validation of urban boundaries derived from global nighttime satellite imagery," International Journal of Remote Sensing, vol. 24, no. 3, pp. 595-609, 2003. 
[23] Z. Liu, C. He, Q. Zhang, Q. Huang, and Y. Yang, "Extracting the dynamics of urban expansion in China using DMSP-OLS nighttime light data from 1992 to 2008," Landscape and Urban Planning, vol. 106, no. 1, pp. 62-72, 2012.

[24] Y. Yang, C. He, Q. Zhang, L. Han, and S. Du, "Timely and accurate national-scale mapping of urban land in China using Defense Meteorological Satellite Program's Operational Linescan System nighttime stable light data," Journal of Applied Remote Sensing, vol. 7, 2013.

[25] X. Cao, J. Chen, H. Imura, and O. Higashi, "A SVM-based method to extract urban areas from DMSP-OLS and SPOT VGT data," Remote Sensing of Environment, vol. 113, no. 10, pp. 2205-2209, 2009.

[26] Y. Xie and Q. Weng, "Updating urban extents with nighttime light imagery by using an object-based thresholding method," Remote Sensing of Environment, vol. 187, pp. 1-13, 2016.

[27] Y. Zhou, S. J. Smith, C. D. Elvidge, K. Zhao, A. Thomson, and M. Imhoff, "A cluster-based method to map urban area from DMSP/OLS nightlights," Remote Sensing of Environment, vol. 147, pp. 173-185, 2014.

[28] Y. Zou, H. Peng, G. Liu, K. Yang, Y. Xie, and Q. Weng, "Monitoring urban clusters expansion in the middle reaches of the Yangtze River, China, using time-series nighttime light images,” Remote Sensing, vol. 9, no. 10, p. 1007, 2017.

[29] A. K. Cherri and M. A. Karim, "Optical symbolic substitution: edge detection using Prewitt, Sobel, and Roberts operators," Applied Optics, vol. 28, no. 21, p. 4644, 1989.

[30] J. R. Dim, T. Takamura, P. Kathri, N. Kikuchi, and T. Y. Nakajima, "Validation of two MODIS aerosols algorithms with SKYNET and prospects for future climate satellites such as the GCOM-C/SGLI," Advances in Meteorology, vol. 2013, Article ID 584816, 8 pages, 2013.

[31] N. Nausheen, A. Seal, P. Khanna, and S. Halder, "A FPGA based implementation of Sobel edge detection," Microprocessors and Microsystems, vol. 56, pp. 84-91, 2018.

[32] O. R. Vincent and O. Folorunso, "A descriptive algorithm for sobel image edge detection," Proceedings of Informing Science \& IT Education Conference (InSITE), vol. 9, 2009.

[33] C. Zhang and J. Fang, "Edge Detection Based on Improved Sobel Operator," Advances in Computer Science Research, vol. 52, 2016.

[34] C. Higurashi, D. Ziskin, K. Baugh et al., "A fifteen year record of global natural gas flaring derived from satellite data," Energies, vol. 2, no. 3, pp. 595-622, 2009.

[35] X. Li and Y. Zhou, "Urban mapping using DMSP/OLS stable night-time light: a review," International Journal of Remote Sensing, vol. 38, no. 21, pp. 6030-6046, 2017.

[36] K. Yi, Y. Zeng, and B. Wu, "Mapping and evaluation the process, pattern and potential of urban growth in China," Applied Geography, vol. 71, pp. 44-55, 2016.

[37] Q. Zhang, B. Li, D. Thau, and R. Moore, "Building a better urban picture: combining day and night remote sensing imagery," Remote Sensing, vol. 7, no. 9, pp. 11887-11913, 2015.

[38] M. Heath, S. Sarkar, T. Sanocki, and K. Bowyer, "Comparison of edge detectors," Computer Vision and Image Understanding, vol. 69, no. 1, pp. 38-54, 1998.

[39] Z. Wang, K. Wang, F. Yang, S. Pan, and Y. Han, "Image segmentation of overlapping leaves based on Chan-Vese model and Sobel operator," Information Processing in Agriculture, vol. 5, no. 1, pp. 1-10, 2018.

[40] C. M. Kyba, T. Kuester, A. Miguel et al., "Artificially lit surface of Earth at night increasing in radiance and extent," Science Advances, vol. 3, 2017.
[41] F. C. Hus, K. Baugh, T. Ghosh, M. Zhizhin, and C. Elvidge, "DMSP-OLS radiance calibrated nighttime lights time series with intercalibration," Remote Sensing, vol. 7, no. 2, pp. 1855-1876, 2015.

[42] T. Ma, Y. Zhou, C. Zhou, S. Haynie, T. Pei, and T. Xu, "Nighttime light derived estimation of spatio-temporal characteristics of urbanization dynamics using DMSP/OLS satellite data," Remote Sensing of Environment, vol. 158, pp. 453-464, 2015.

[43] K. Baugh, C. Elvidge, T. Ghosh, and D. Ziskin, "Development of a 2009 stable lights product using DMSP-OLS data," Proceedings of the Asia-Pacific Advanced Network, vol. 30, 2010. 